\title{
Standounkt
}

Klimaschutz und Nachhaltige Entwicklung

\section{Die unzulängliche Architektur des Clean Development Mechanism}

\begin{abstract}
Emissionszertifikate machen Kohlenstoffdioxid-Einsparungen käuflich und sollen nachhaltige Entwicklung im globalen Süden fördern. Die Rechnung geht oft nicht auf. Es gibt in der Architektur des Clean Development Mechanism keine Anreize, Projekte mit einem Beitrag zur nachhaltigen Entwicklung umzusetzen. Stattdessen boomen Projekte an Industrieanlagen. Von Nataly Jürges
\end{abstract}

$\mathbf{F}_{\mathrm{i}}^{\mathrm{i}}$ egen ist einer der größten Klimakiler, der mit dem westlichen Lebensstil verbunden ist. Ein gutes Klima-Gewissen ist trotz Fernreise vergleichsweise preiswert zu haben, denn verschiedene Organisationen bieten den Kauf von Zertifikaten an, durch die die Flugreise klimatisch neutralisiert wird. Diese Idee, den Kauf von Emissionseinsparungen zu ermöglichen, ist in internationalen Verträgen verankert: Als Teil des Kyoto-Protokolls macht der Clean Development Mechanism (CDM) den internationalen Handel mit Emissionszertifikaten von Kohlenstoffdioxid $\left(\mathrm{CO}_{2}\right)$ seit 2005 möglich.

\section{Fehlende Anreize}

Einige der Zertifikate stammen beispielsweise aus einem EnergieeffizienzProjekt, in dem holzsparende Herde an nigerianische Haushalte subventioniert verkauft werden. Durch die Verbreitung der Herde werden Emissionen gesenkt und Entwaldung reduziert. Das Projekt zeigt die gelungene Kombination nachhaltiger Entwicklung und Klimaschutz (1). Es entspricht einer Sonderkategorie von CDM-Projekten, den sogenannten Kleinprojekten. Diese leisten wegen ihres dezentralen Charakters meist einen hohen Beitrag zur nachhaltigen Entwicklung. Die Kosten für die Anerkennung als CDM-Projekt fallen in Kleinprojekten vergleichsweise viel stärker ins Gewicht als in Projekten an Industrieanlagen, da sie fast unabhängig von der Höhe der Emissionsminderung sind. Stattdessen sind
Projekte im Industriebereich deutlich profitabler (Sterk 2004). Die lokale Bevölkerung hat von Projekten an Industrieanlagen meist nichts.

Auch wenn als Nebenziel die nachhaltige Entwicklung des Gastlandes gefördert werden soll, gibt es in der CDM-Architektur keine echten Anreize dies umzusetzen. Stattdessen sind Projekte im Industriebereich deutlich profitabler. In der Ausgestaltung des CDM wurden Transaktionskosten zu wenig berücksichtigt, um das Nebenziel der nachhaltigen Entwicklung im Gastland zu erreichen. Zwar gibt es ein vereinfachtes Genehmigungsverfahren für Kleinprojekte, die administrativen Kosten bleiben aber trotzdem im Vergleich zu Großprojekten unverhältnismäßig hoch (Sterk 2004).

\section{Klimapolitische Perspektiven}

Die Zukunft der globalen Klimapolitik nach Ende der Verpflichtungsperiode des Kyotoabkommens im Jahr 2012 ist unsicher. Daher besteht aktuell die Gelegenheit durch eine gezielte Änderung der CDM-Richtlinien Projekte mit einem hohen Entwicklungsnutzen zu stärken.

Rund 1,2 Milliarden Menschen leben derzeit von weniger als einem US-Dollar pro Tag. Diese Menschen wollen ihren Konsum deutlich steigern. Aber ist es überhaupt möglich, eine angemessene Entwicklung zu erzielen, wenn die Emissionen dabei nicht das Niveau des globalen Nordens erreichen dürfen? Daher ist es eine zentrale Herausforderung der internationalen Klimapolitik einen Weg zu finden, wie Armutsüberwindung und Klimaschutz besser als bisher kombiniert werden können (Santarius 2008). Mit dem CDM hat bereits der Versuch begonnen, Armutsüberwindung und Klimaschutz miteinander zu vereinen, leider bislang nur mit mäßigem Erfolg. Es gibt zu wenige Projekte, die einen echten Beitrag zur nachhaltigen Entwicklung leisten. Die Inkaufnahme von, ökonomisch betrachtet, weniger effizienten Wegen der Emissionsreduktion zugunsten einer deutlichen Steigerung des Beitrages zu nachhaltigen Entwicklung ist von wesentlicher Bedeutung dafür, dauerhaft einen tragfähigen klimapolitischen Konsens zwischen Industrieländern und Entwicklungsstaaten zu ermöglichen.

Die Idee CDM hat das Potenzial langfristig doch noch zum Erfolgskonzept zu werden. Globaler Klimaschutz kann nur erfolgreich sein, wenn es den Staaten der Erde gelingt, sich auf eine gemeinsame Strategie $\mathrm{zu}$ einigen. Um dies $\mathrm{zu}$ erreichen, muss die internationale Klimapolitik stärker als bisher Armutsminderung im globalen Süden in ihre Agenda aufnehmen.

\section{Anmerkung \\ (1) Mehr über das Projekt im Internet: http://www.nigeria.I-h-I.org; http://www.atmosfair.de.}

\section{Literatur}

Santarius, T.: Fairhandeln im Treibhaus. In: Altvater, E. / Brunnengräber, A. (Hrsg.): Ablasshandel gegen Klimawandel? Marktbasierte Instrumente in der globalen Klimapolitik und ihre Alternativen. VSA-Verlag. Hamburg 2008. S. 119-133.

Sterk, W.: CDM-Projekte - Neue Wege für die entwicklungspolitische Arbeit lokaler Initiativen in Deutschland? Policy Paper 2/2004. Internet: http://www.jiko-bmu.de/files/basisinformationen/application/pdf/pp_02-04-ssc-s.pdf

I AUTORIN + KONTAKT

Nataly Jürges ist wissenschaftliche Mitarbeiterin am Johann Heinrich von Thünen-Institut im Institut für Ländliche Räume.

Nataly Jürges, Johann Heinrich von Thünen-Institut, Bundesforschungsinstitut für ländliche Räume, Wald und Fischerei - Institut für Ländliche Räume, Bundesallee 50, 38116 Braunschweig. Tel.: +495315965223, E-Mail: nataly.juerges@vti.bund.de 


\section{Lizenzhinweis}

Die Beiträge in ÖkologischesWirtschaften werden unter der Creative-Commons-Lizenz "CC 4.0 Attribution Non-Commercial No Derivatives" veröffentlicht. Im Rahmen dieser Lizenz muss der Autor/Urheber stets genannt werden, das Werk darf nicht bearbeitet, abgewandelt oder in anderer Weise verändert und außerdem nicht kommerziell genutzt werden. Die digitale Version des Artikels bleibt für zwei Jahre Abonnent/innen vorbehalten und ist danach im Open Access verfügbar. 